\title{
Strong ground motion observation and estimation: From predictive relationships and modelling to real-time shakemaps
}

\author{
Suhadolc Peter*, Costa Giovanni and Moratto Luca
}

Department of Earth Sciences, University of Trieste, Via Weiss 1, 34127 Trieste, ITALY

* For correspondence, email: suhadolc@units.it

After a brief review on strong ground motion observation and estimation in continental convergence areas, predictive relationships are discussed with a special emphasis on the nearsource areas of large earthquakes. A comparison with relationships derived for low-magnitude events is made and it is demonstrated that the latter cannot be used to extrapolate the ground motion level for large-magnitude events.

The acceleration at low frequencies can be successfully estimated through a deterministic modeling, given an appropriate knowledge about the velocity structure and earthquake source parameters. In particular, the question of the appropriateness of the velocity model is addressed. A series of tests to evaluate how well different 1D approximations of a 2D structural model reproduce the ground motion, in particular its peak amplitude, is discussed. The deterministic modeling can produce groundshaking synthetic maps, or scenarios. Examples for some important past seismic events in the Southern Alps area are discussed.

Finally, the strong ground motion level can nowadays be addressed with ShakeMaps, that are generated within few minutes from the earthquake occurrence. They are based on the TriNet "ShakeMap" software of Wald et al. (1999) and interfaced with a real-time acquisition system, that retrieves the real-time waveforms and posts results automatically on the web page. For the calibration of the Shake Maps regional model in the Southern Alps area a simple geological classification scheme and two ground-motion relations for different magnitude ranges are used. The system has been successfully tested for medium to moderate events $(\mathrm{ML}<4.5)$. 